\title{
High-sensitivity rod photoreceptor input to the blue- yellow color opponent pathway in macaque retina
}

\author{
Greg D Field ${ }^{1}$, Martin Greschner ${ }^{1}$, Jeffrey L Gauthier ${ }^{1}$, Carolina Rangel ${ }^{2}$, Jonathon Shlens ${ }^{1,3}$, Alexander Sher ${ }^{4}$, \\ David W Marshak ${ }^{2}$, Alan M Litke ${ }^{4} \&$ EJ Chichilnisky $^{1}$
}

\begin{abstract}
Small bistratified cells (SBCs) in the primate retina carry a major blue-yellow opponent signal to the brain. We found that SBCs also carry signals from rod photoreceptors, with the same sign as S cone input. SBCs exhibited robust responses under low scotopic conditions. Physiological and anatomical experiments indicated that this rod input arose from the All amacrine cell-mediated rod pathway. Rod and cone signals were both present in SBCs at mesopic light levels. These findings have three implications. First, more retinal circuits may multiplex rod and cone signals than were previously thought to, efficiently exploiting the limited number of optic nerve fibers. Second, signals from All amacrine cells may diverge to most or all of the $\sim 20$ retinal ganglion cell types in the peripheral primate retina. Third, rod input to SBCs may be the substrate for behavioral biases toward perception of blue at mesopic light levels.
\end{abstract}

The mammalian retina contains $\sim 20$ morphologically distinct retinal ganglion cell (RGC) types ${ }^{1-3}$. Each RGC type receives input from a distinct set of retinal interneuron types, encodes a different aspect of the visual scene, and projects to a distinct set of targets in the brain. Among the RGC types in the primate retina, small bistratified cells (SBCs) are of particular interest; they display cone opponent responses appropriate for blue-yellow color vision ${ }^{4}$, they are the fifth most numerous RGC type in the primate ${ }^{2}$ and they form a major projection to the koniocellular layers of the lateral geniculate nucleus $(\mathrm{LGN})^{5}$.

It is currently unknown whether SBCs contribute to scotopic (rod mediated) vision in addition to their role in photopic (cone mediated) vision. The possibility that SBCs carry rod signals is suggested by psychophysical studies demonstrating perceptual shifts toward blue hues in mesopic (rod and cone mediated) vision ${ }^{6}$. However, previous measurements from blue-yellow color opponent cells in the primate retina have revealed little or no input from rods ${ }^{7}$, and recordings from possible SBC target neurons in the LGN have produced mixed results ${ }^{8,9}$.

Rod input to SBCs has potentially important implications for the function and organization of parallel pathways in the primate visual system. If SBCs avoid rod input, it would indicate that a substantial fraction $(\sim 10 \%)^{2}$ of the axons in the optic nerve carry no behaviorally relevant signal during night vision. It would also indicate that one function of parallel pathway organization is to specialize certain RGC types for a limited range of light levels. Furthermore, it would suggest that the presynaptic circuitry of SBCs avoids two kinds of rod inputs: gap junctions between rods and $\mathrm{S}$ cones and gap junctions between AII amacrine cells and S cone bipolar cells. Conversely, if SBCs do receive rod input, then this input may help to explain perceptual biases toward blue hues under mesopic conditions. It would also suggest that the visual system multiplexes rod and cone signals in each RGC to use the optic nerve efficiently.

We used large-scale multi-electrode recordings ${ }^{10}$ from peripheral primate retina to test whether SBCs receive rod input. This approach allowed for long, stable recordings from identified SBCs, an important technical advantage. We recorded from nearly complete populations of SBCs with receptive fields that collectively covered the recorded region of retina ${ }^{11}$. SBCs received rod input with the same on type response polarity as $\mathrm{S}$ cone input. SBC receptive fields were substantially larger at rod-dominated light levels, revealing a change in spatial processing in night versus day vision. Furthermore, physiological and anatomical experiments indicated that rod signals reach SBCs via a known highsensitivity pathway; rod signals are conveyed by rod-specific bipolar cells to AII amacrine cells, which in turn form gap junctions with ON cone bipolar cells that provide excitatory input to RGCs ${ }^{12,13}$.

\section{RESULTS}

To test for rod input to SBCs, we recorded from RGCs in peripheral primate retina with a 512-electrode array at two light levels (Fig. 1): a high light level at which retinal signaling was dominated by cone photoreceptors (photopic, $\sim 1,000$ photo-isomerizations $\left(\mathrm{P}^{\star}\right)$ per cone per s) and a low light level at which retinal signaling was dominated by rod photoreceptors (scotopic, $\sim 1.0 \mathrm{P}^{\star}$ per rod per s). Receptive fields were estimated by computing the spike triggered average (STA) of a white-noise stimulus presented at each light level (see Online Methods).

\footnotetext{
${ }^{1}$ Salk Institute for Biological Studies, La Jolla, California, USA. ${ }^{2}$ Department of Neurobiology and Anatomy, University of Texas Medical School, Houston, Texas, USA. ${ }^{3}$ University of California, Berkeley, California, USA. ${ }^{4}$ Santa Cruz Institute for Particle Physics, University of California, Santa Cruz, California, USA. Correspondence should be addressed to G.D.F. (gfield@salk.edu).
}

Received 8 April; accepted 29 May; published online 9 August 2009; doi:10.1038/nn.2353 

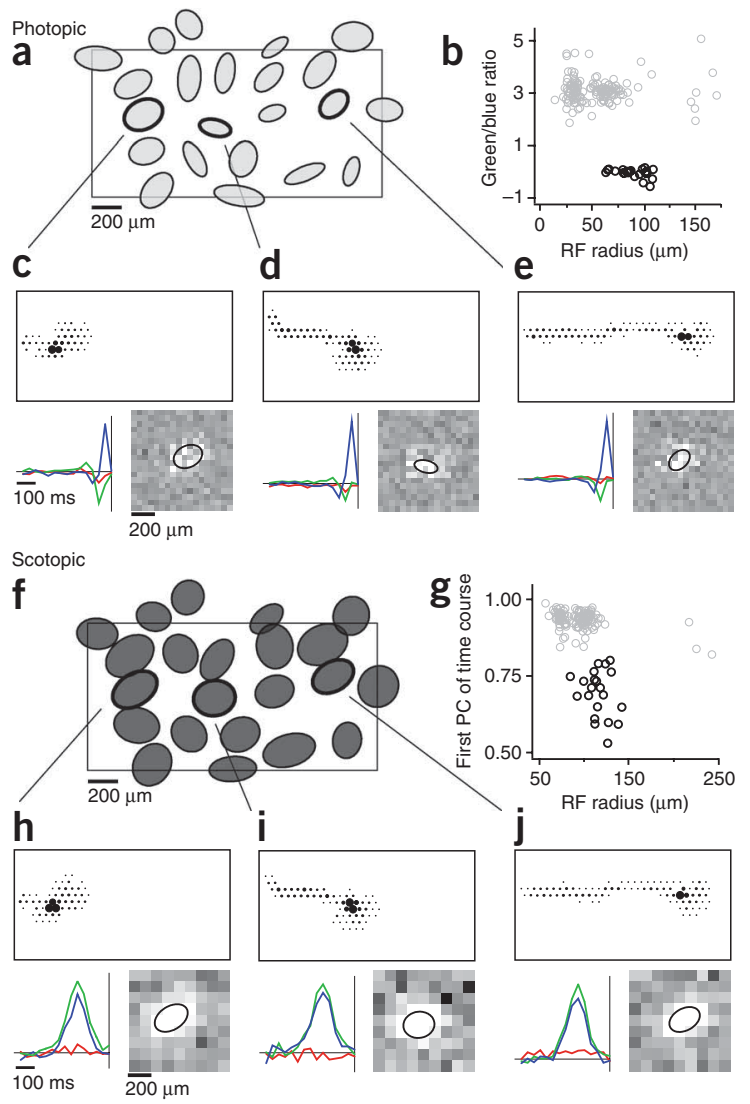

SBCs were identified at the high light level by observing a functional class of RGCs with blue-ON/yellow-OFF receptive fields that formed a mosaic uniformly covering the retina (Fig. 1a,b), with a density corresponding to that of the morphologically defined small bistratified type $^{11}$. Blue-ON/yellow-OFF responses were identified by increments in the blue display primary and decrements in the red and green primaries preceding spikes (Fig. 1c-e). Such responses are consistent with an ON response mediated by $\mathrm{S}$ cones and an off response mediated by $\mathrm{L}$ and M cones ${ }^{11,14}$.

Successful tracking of SBCs between the high and low light levels was confirmed by two independent observations. First, a functional classification of RGCs recorded at the low light level (Fig. 1g) produced a class

Figure 2 Change in SBC receptive field size between scotopic and photopic light levels. (a) Overlaid rod-mediated (dark gray) and S cone-mediated (light gray) receptive fields from four recordings. Retina 1 is the same retina used for Figure 1. The rod-mediated receptive fields for retinas 1-3 were measured at $1.2 \mathrm{P}^{*}$ per rod per $\mathrm{s}$, and those for retina 4 were measured at $0.077 \mathrm{P}^{*}$ per rod per $\mathrm{s}$. All cone-mediated receptive fields were measured at $\sim 800 \mathrm{P}^{*}$ per cone per $\mathrm{s}$. Rectangles indicate the outline of the electrode array. (b) Comparison of receptive field size (radius of circle with area equal to ellipse) between rod- and cone-dominated conditions in a single preparation. (c) Comparison of the mean receptive field radius in eight preparations. Circles and squares represent preparations in which the scotopic light level was 1.2 and $0.077 \mathrm{P}^{*}$ per rod per s, respectively. Triangles represent preparations in which the pigment epithelium remained attached to the retina (see Online Methods) and the scotopic light level was $1.5 \mathrm{P} *$ per rod per s. Error bars represent s.d. (d) Reversibility of change in receptive field size. Receptive field size was averaged across 17 (filled circles) and 15 (open circles) SBCs in two preparations with the pigment epithelium attached to the retina. The light level was changed from cone $\left(\sim 1,000 \mathrm{P}^{*}\right.$ per cone per s) to rod $\left(1.5 \mathrm{P}^{*}\right.$ per rod per s) and back to cone light levels. Error bars represent \pm s.e.m.
Figure $1 \mathrm{SBC}$ identification at photopic and scotopic light levels. (a) Spatial receptive fields of 22 simultaneously recorded SBCs at $\sim 800 \mathrm{P}^{*}$ per cone per s (see Online Methods). Ellipses represent the 1-s.d. contour of a fit to the blue-on receptive field (see Methods). Rectangle indicates the outline of the electrode array $(1,800 \times 900 \mu \mathrm{m})$. (b) Scatter plot shows the classification of SBCs (black circles) that distinguished them from all other on RGCs (gray circles, see Online Methods). (c-e) Three cells are highlighted from the receptive field mosaic in a. Top, the electrophysiological image (see Online Methods). Bottom left, STA time course for each display primary (red, green and blue). The abscissa indicates the time to the spike and the ordinate indicates the primary intensities relative to background (arbitrary units, a.u.). Bottom right, spatial profile of the STA for the blue primary. The ellipse represents the 1-s.d. contour from fit. (f) Spatial receptive fields of the cells in a estimated at $1.2 \mathrm{P}^{*}$ per rod per $\mathrm{s}$. (g) Classification of SBCs at the low light level (black circles, see Online Methods).

$(\mathbf{h}-\mathbf{j})$ Electrophysiological images (top) and STA time courses and spatial profiles (bottom) measured at the low light level for the cells shown in c-e. Spatial profiles are from the sum of the blue and green primaries in the STA.

with a nearly identical spatial organization of receptive fields (Fig. 1a,f). Second, neurons at the low light level with corresponding receptive field locations exhibited nearly identical electrophysiological images (Fig. 1c-e,h-j). The electrophysiological image is the spike-triggered average spatiotemporal pattern of electrical activity elicited by an identified cell, which provides a unique electrical footprint for each RGC ${ }^{10}$ (see Online Methods). Thus, SBCs were tracked across light levels.

\section{SBCs receive cone and rod input}

The high and low light levels revealed cone and rod input to SBCs, respectively. The high light level produced a photon flux corresponding to $\sim 100 \mathrm{Td}$, well above the value that saturates the rods ${ }^{15}$. Furthermore, the blue-ON/yellow-OFF opponent response displayed by the SBCs could not be mediated solely by the rods (Fig. 1c-e). Thus, light responses in SBCs at the high light level were primarily or exclusively mediated by cones.

On the other hand, the low light level produced a photon flux that corresponded to $\sim 0.1$ scotopic $\mathrm{Td}$, well below the psychophysically determined cone threshold of $\sim 10$ scotopic $\mathrm{Td}^{16}$. Consistent with rod input at the low light level, the time course of the SBC light response was more monophasic and the time to peak was $4.1 \pm 0.6$-fold (mean \pm s.d.) longer than at the high light level ${ }^{7,17,18}$. Furthermore, the STAs of the SBCs at the low light level did not exhibit a color opponent response (Fig. 1h-j). Instead, they exhibited a relative sensitivity to the red, green

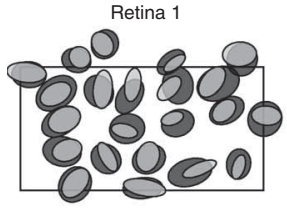

Retina 3

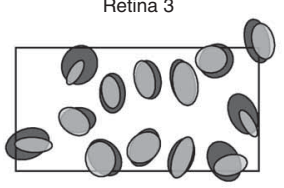

$\square$ Photopic b
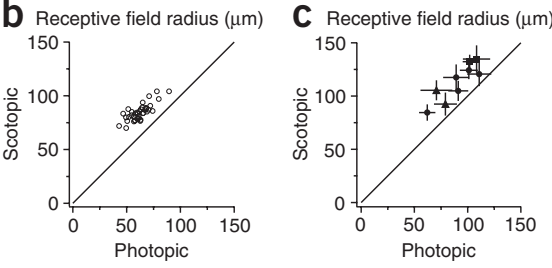

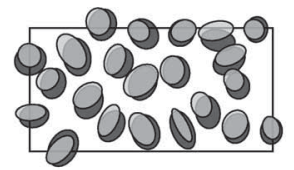

Retina 4
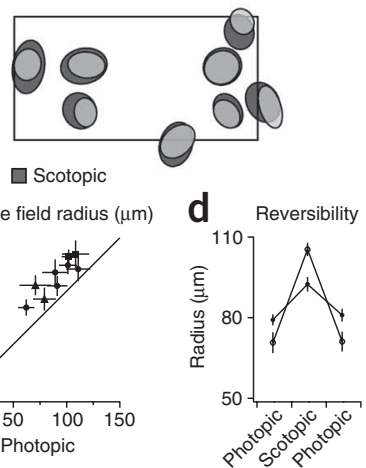
a
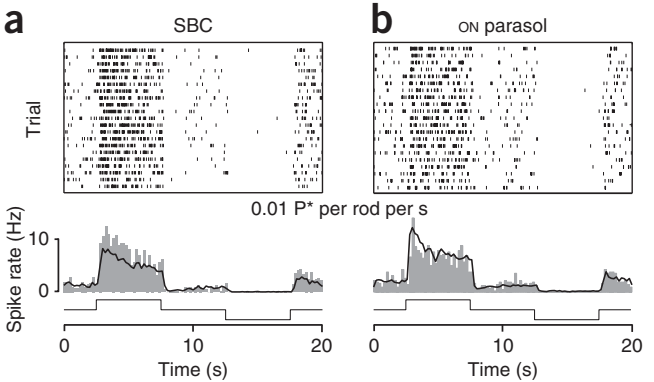

Figure 3 Responses of SBCs and on parasol cells to dim light steps. (a) Top, spike rasters from a single SBC in response to 20 repetitions of full field light steps that changed every $5 \mathrm{~s}$ from gray $\left(0.01 \mathrm{P}^{*}\right.$ per rod per $\left.\mathrm{s}\right)$ to white $\left(0.02 \mathrm{P}^{*}\right.$ per rod per $\left.\mathrm{s}\right)$ to gray to nominal black $\left(0.00008 \mathrm{P}^{*}\right.$ per rod per s). Middle, peristimulus time histogram (PSTH, bin size $=0.2 \mathrm{~s}$ ) of the response of an individual SBC (gray bars) and the average from six simultaneously recorded SBCs (black trace). Bottom, time course of the stimulus. (b) Top, spike raster from a single on parasol cell recorded simultaneously with the SBC in a. Middle, PSTH of the on parasol cell (gray bars) and average from 23 on parasol cells recorded simultaneously. Bottom, time course of stimulus.

and blue display primaries $(0.074 \pm 0.05,1.0$ and $0.83 \pm 0.04$ (s.e.m.), respectively, normalized by the green primary), consistent with the values predicted from the spectral sensitivity of rods $(0.070,1.0$ and 0.84 ). Finally, simultaneously recorded on and off parasol cells exhibited nearly identical relative sensitivities to the display primaries at the low light level (on parasol: $0.073 \pm 0.005,1.0$ and $0.82 \pm 0.004$; off parasol: $0.10 \pm 0.01,1.0$ and $0.84 \pm 0.01$ for red, green and blue, respectively), further supporting the interpretation that rod signals provided the dominant or exclusive input at the low light level.

\section{SBC receptive fields are larger under scotopic conditions}

Previous studies of other RGC types have indicated that receptive field sizes are larger under scotopic than photopic conditions ${ }^{18-20}$. A comparison of receptive field sizes between the low and high light levels revealed a similar result in SBCs (Fig. 2). For most cells, the rodmediated receptive field was larger than the cone-mediated receptive field; this trend was observed across all eight recordings examined (Fig. 2b,c). On average, the rod-mediated receptive fields were $\sim 20 \%$ larger in diameter than the cone-mediated receptive fields of the same cells. Note that we observed no receptive field surround at the low light level, which is also consistent with observations from other RGC types ${ }^{19,21}$ (but see ref. 20).

We tested whether the dependence of receptive field size on light level was reversible by recording from retinas with the pigment epithelium attached (see Online Methods). This allowed the photopigment to efficiently regenerate when switching between photopic and scotopic conditions. In both of the preparations tested (32 SBCs), the change in receptive field size was reversible (Fig. 2 d).

Figure 4 L-APB nearly eliminated the light response in off parasol cells at low scotopic light levels. (a) Top, spike rasters from a single off parasol cell in response to 20 repetitions of full-field light steps that changed every $3 \mathrm{~s}$ from gray $\left(0.14 P^{*}\right.$ per rod per $\left.s\right)$ to white $\left(0.28 P^{*}\right.$ per rod per $\left.s\right)$ to gray to nominal black $\left(0.0005 \mathrm{P}^{*}\right.$ per rod per $\left.\mathrm{s}\right)$. Bottom, PSTH (bin size $=0.1 \mathrm{~s}$ ) of the off parasol cell (gray bars) and the average from 24 simultaneously recorded off parasol cells (black trace). (b) The individual cell and population of cells shown in a with bath application of $10 \mu \mathrm{M}$ L-APB. (c) The same individual and population of cells shown in a stimulated at light levels tenfold higher (gray $=1.4 \mathrm{P}^{*}$ per rod per $\mathrm{s}$, white $=2.8 \mathrm{P}^{*}$ per rod per $\mathrm{s}$ and black $=0.005 \mathrm{P}^{*}$ per rod per s). (d) Same as c with $10 \mu \mathrm{M}$ L-APB.

\section{Rod responses persist in SBCs in low scotopic conditions}

The presence of robust rod-driven responses at light levels of $\sim 1.0 \mathrm{P}^{*}$ per rod per s described above suggests that the high-sensitivity rod pathway mediated by AII amacrine cells contributes signals to the presynaptic circuitry of $\mathrm{SBCs}^{13,16,22}$. This pathway is thought to dominate visual signaling under low scotopic conditions. However, recent recordings from primate cones ${ }^{23}$ and mouse $\mathrm{RGCs}^{24}$ suggest that gap junctions between rods and cones could also provide reliable rod signals at $\sim 1.0 \mathrm{P}^{\star}$ per rod per s.

Further evidence for the involvement of the AII pathway was provided by the fact that SBCs exhibited light responses substantially below previously measured thresholds for the rod-to-cone gap-junction pathway ${ }^{23,24}$. First, white-noise stimulation at $<0.1 \mathrm{P}^{\star}$ per rod per $\mathrm{s}$ elicited clear light responses in SBCs in two preparations (Fig. 2a,c). Second, SBCs responded robustly to a stimulus consisting of light steps about a mean light level of $0.01 \mathrm{P}^{\star}$ per rod per s (Fig. 3a). Furthermore, these responses were similar to those produced by simultaneously recorded on parasol cells (Fig. 3b), which project to the magnocellular layers of the LGN and exhibit high contrast sensitivity under both photopic and scotopic conditions. The fact that high-sensitivity light responses and mosaics of SBC receptive fields were recorded at these low light levels supports the hypothesis that the AII amacrine cell pathway provides rod input to SBCs.

\section{L-APB blocks responses at light levels $<1 \mathrm{P}^{*}$ per rod per s}

To further test whether AII amacrine cells mediate the observed rod input to SBCs, we measured the responses of off parasol cells to spatially uniform light steps with and without bath application of $10 \mu \mathrm{M}$ L-APB (L-(+)-2-amino-4-phosphonobutyric acid; Fig. 4). L-APB is a group III metabotropic glutamate receptor agonist that saturates the postsynaptic receptors (mGluR6) of on type bipolar cells, effectively blocking synaptic transmission between photoreceptors and all on type (including rod) bipolar cells ${ }^{25}$. If rod signals are conveyed to RGCs exclusively by the rod bipolar-AII amacrine cell circuit, then L-APB should block the light response of off type RGCs, in addition to its typical effect of blocking light responses in on type RGCs ${ }^{26}$. Alternatively, to the extent that rod signals are conveyed to cones via gap junctions, responses in off type RGCs should not be blocked by L-APB, as rod signals will be conveyed to off type RGCs via synapses using ionotropic glutamate receptors, which are insensitive to L-APB.

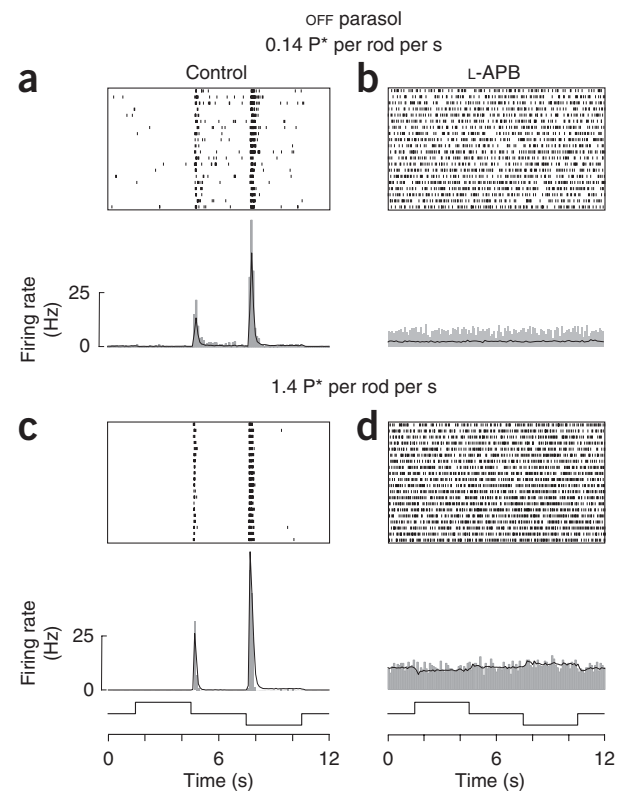




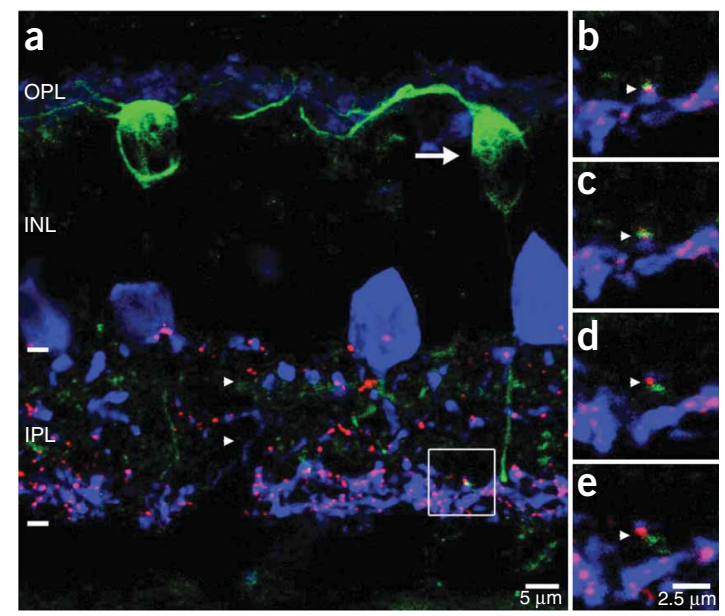

OFF parasol cells exhibited robust responses to light steps about a mean light level of $0.14 \mathrm{P}^{\star}$ per rod per s (Fig. 4a). These responses were eliminated by L-APB (Fig. $\mathbf{4 b}$ ). Note that light responses were undetectable despite averaging over 20 cycles of the step stimulus from 24 simultaneously recorded off parasol cells (Fig. 4b). Responses to light steps about a mean light level that was tenfold higher $\left(1.4 \mathrm{P}^{\star}\right.$ per rod per s) were also reduced by L-APB (Fig. 4c,d). At this higher light level, however, averaging across trials and cells revealed a weak response modulation (Fig. 4d). At both light levels, the light response recovered when L-APB was washed from the bath (data not shown). As expected, the responses of SBCs and oN parasol cells were also eliminated by L-APB at both light levels (data not shown). In the presence of $\mathrm{L}-\mathrm{APB}$, the maintained spike rate increased for off parasol cells and was zero for both SBCs and on parasol cells (data not shown), consistent with previous studies ${ }^{26}$. These results indicate that light responses to stimuli generating fewer than $\sim 1-2 \mathrm{P}^{\star}$ per rod per $\mathrm{s}$ are mediated predominantly, or exclusively, by the AII amacrine cell pathway, consistent with previous observations in mouse ${ }^{27}$. However, the small L-APB-insensitive rod signals at the higher light level suggest an increasing contribution of rod-cone gap junctions as the light level is increased (see Discussion).

\section{Gap junctions between All amacrine and S cone bipolar}

The above results suggest the presence of gap junctions between AII amacrine cells and S cone bipolar cells, which provide the oN type input to SBCs. However, previous studies have suggested that AII amacrine cells may avoid forming gap junctions with $\mathrm{S}$ cone bipolar cells ${ }^{28}$. Therefore, we searched for such contacts by immunolabeling S cone bipolar cells, AII amacrine cells and the connexin 36 protein, which forms the gap junction between AII amacrine cells and other on-cone bipolar cells ${ }^{24}$.

We used the glycine-extended gastrin-cholecystokinin precursor, G6-gly, to label S cone bipolar cells (Fig. 5) ${ }^{29}$. Antibodies to calretinin were used to label AII amacrine cells ${ }^{30}$. Cell types were identified on the basis of morphological characteristics (see Supplementary Methods). Antibodies to connexin 36 (red) were used to label putative gap junctions in the inner plexiform layer ${ }^{31}$.

In stratum 5 of the inner plexiform layer, appositions were observed between $\mathrm{S}$ cone bipolar cell axon terminals and dendrites of AII amacrine cells, and puncta containing immunoreactive connexin 36 were found at these sites (Fig. 5a). These contacts were followed through consecutive optical sections $(0.5 \mu \mathrm{m}$ steps $)$ to avoid misinterpretation as a result of superposition of nonserial image planes (Fig. 5b-e). Similar contacts were observed in the retinas of all three
Figure 5 Gap junctions are present at appositions between All amacrine and $\mathrm{S}$ cone bipolar cells. (a) A stack of five optical sections of triple-labeled macaque retina containing two $S$ cone bipolar cells labeled with antibody to G6-gly (green), four All amacrine cells labeled with antibody to calretinin (blue) and numerous connexin 36-immunoreactive puncta (red). The large arrow indicates the cell body of an S cone bipolar cell. Note the characteristic, laterally oriented dendrites in the outer plexiform layer and the axon descending to stratum 5 of the inner plexiform layer. The small arrowheads indicate the strata containing labeled amacrine cell dendrites. One of the labeled S cone bipolar cell axon terminals contacts an All amacrine cell dendrite and a labeled punctum is present at the site (square). (b-e) Consecutive single optical sections of the region indicated by the square in $\mathbf{a}$ are shown at higher magnification. Arrowheads in $\mathbf{b}-\mathbf{e}$ identify the contact location. INL, inner nuclear layer; IPL, inner plexiform layer; OPL, outer plexiform layer.

macaques (Supplementary Figs. 1-4). Thus, AII amacrine cells appear to make gap junctions with $\mathrm{S}$ cone bipolar cells.

\section{Simultaneous rod and cone input to SBCs}

The observed rod input to SBCs under low scotopic conditions suggests that rod and cone signals may mix at mesopic light levels. Alternatively, the mechanisms carrying rod signals to SBCs could saturate before reaching the threshold for cone activation, preventing mixing of rod and cone signals. We tested for simultaneous rod and cone input by measuring SBC light responses across a range of light levels (Fig. 6). At the highest light levels, SBCs exhibited a relative sensitivity to the red, green and blue display primaries that was consistent with a S-ON/ $(\mathrm{L}+\mathrm{M})$-ofF cone-mediated response ${ }^{11,14}$. However, between 150 and $300 \mathrm{P}^{\star}$ per rod per s, SBCs exhibited a marked change in spectral tuning: increments, rather than decrements, in the green display primary tended to precede spikes.

In principle, this change in spectral tuning could be explained two ways: a weakening of the ( $\mathrm{L}+\mathrm{M})$-off surround and/or the inclusion of rod input with the same sign as $\mathrm{S}$ cone input. In the former case, the sensitivity to the green display primary should approach the value predicted from the spectral sensitivity of the $S$ cones. The expected sensitivity to the green relative to blue display primary for $S$ cones was 0.11 (see Online Methods). At $150 \mathrm{P}^{\star}$ per rod per s, however, the observed ratio was $0.35 \pm 0.04$. Thus, the large sensitivity to increments of the green primary implies rod input to the SBCs. Cones must also provide input to SBCs at $150 \mathrm{P}^{\star}$ per rod per $s$ because the response remained color opponent (off type response to modulation of the red primary) and the spectral tuning of the response did not match that expected from rods (STA time course at $1.5 \mathrm{P}^{\star}$ per rod per s; Fig. 6). At progressively lower mean light levels, the relative sensitivity to the three display primaries shifted to the values expected from pure rod input. Therefore, rod and cone signals mix in SBCs at light levels between $\sim 75$ and $\sim 300 \mathrm{P}^{\star}$ per rod per s.

\section{DISCUSSION}

Our results demonstrate that visual encoding in SBCs extends to low scotopic conditions, that SBC receptive fields are larger under these conditions and that AII amacrine cells convey rod input to the presynaptic circuitry of SBCs. These results have implications for retinal circuitry, night vision and color perception.

\section{The circuitry of rod input to SBCs}

The AII amacrine cell rod pathway (rod to rod bipolar to AII amacrine to cone bipolar to RGC) mediates visual processing near absolute threshold and throughout low scotopic vision ${ }^{12,13}$. The present results indicate that this pathway contributes rod signals to SBCs, the major blue-on/yellow-OFF pathway of the primate visual system. 
Figure 6 Spectral tuning and dynamics of SBC responses depended on light level. From a single recording of 33 SBCs, the mean STA time courses of the SBCs for the red, green and blue display primaries are shown at seven light levels. The light level increases from left to right. At 3,000 P* per rod per $\mathrm{s}$, the rods were saturated and the response was mediated by the cones. Photo-isomerization rates for the $L, M$ and $S$ cones were 1,400, 1,200 and $430 \mathrm{P}^{*}$ per cone per s, respectively. The SBCs exhibited color opponency; increments in the blue display primary and decrements in the red and green display primaries tended to precede spikes. At 150 P* per rod per $\mathrm{s}$, the spectral tuning of the SBCs exhibited a marked change; the SBCs continued to display color opponent responses, but increments, rather than decrements, in the green display primary tended to precede spikes. At $1.5 \mathrm{P}^{*}$ per rod per $\mathrm{s}$, the light level was below cone threshold and the spectral tuning of the response was non-opponent, reflecting pure rod input to the SBCs.

SBCs produced vigorous responses to light steps (Fig. 3) and whitenoise stimulation (Fig. 2) at light levels at which L-APB blocked the response of OFF parasol cells (Fig. 4), suggesting that the AII amacrine cell pathway is the dominant pathway for rod signals reaching RGCs at these light levels. Furthermore, human psychophysical and electroretinogram studies suggest that the rod-cone gap junction pathway is not strongly activated at light levels of $<1$ scotopic $\mathrm{Td}^{16}$, which corresponds to $\sim 10 \mathrm{P}^{\star}$ per rod per $\mathrm{s}^{32}$. Finally, the present results indicate that connexin-36 is present at appositions of AII amacrine cell dendrites and S cone bipolar cell axon terminals (Fig. 5 and Supplementary Figs. 1-4), providing anatomical evidence for the existence of this pathway.

However, these results do not rule out the possibility that gap junctions between rods and S cones contribute rod signals to SBCs at light levels of $>1-2 \mathrm{P}^{\star}$ per rod per s. Indeed, gap junctions between rods and $\mathrm{S}$ cones have been observed in anatomical studies of primate retina (personal communication, S. Massey, University of Texas Health Science Center at Houston). Also in primates, rod activation hyperpolarizes $\mathrm{S}$ cones, presumably through gap junctions (personal communication, J. Schnapf, University of California, San Francisco). The relative contribution of rod-to-cone gap junctions and the AII amacrine cell pathway at mesopic and high scotopic light levels remains unclear.

\section{Comparison with previous studies}

Previous studies of rod input to blue-yellow opponent cells in the primate visual system produced mixed results. One study of the LGN identified some rod input ${ }^{9}$, whereas other studies of the LGN and the retina revealed little or no rod input ${ }^{7,8}$. There are several possible technical aspects of previous studies that could produce discrepant results: insufficient time for the dark adaptation that could cause rod input to be underestimated, the challenge of maintaining stable recordings for long periods of time in vivo, recordings near the fovea where rods are sparse, and recordings from unidentified morphological cell types which could produce variable results if some blue-ON/yellowofF cell types receive rod input while others do not. These potential issues were mitigated in the present study. First, the retina was dark-adapted for $>40$ min before recordings were made under scotopic conditions (see Online Methods). Second, the use of a large-scale multielectrode array provided recordings for 5-15 h from collections of cells in which the stability could be confirmed by electrophysiological images of individual cells and the position of individual receptive fields in a mosaic of other receptive fields with matched response properties. Third, recordings were made from peripheral primate retina, where rod density is high and relatively uniform ${ }^{15}$. Fourth, the observation of nearly complete receptive field mosaics permitted unambiguous identification of a single morphologically defined cell type, the $\mathrm{SBC}^{11}$.

Gap junctions between AII amacrine cells and S cone bipolar cells were not observed in two earlier electron microscopic studies, probably for technical reasons. In one study, $S$ cone bipolar cells in peripheral retina were filled with an electron-dense peroxidase reaction product, which would make detection of gap junctions difficult, and the sample was limited as a result of the low spatial density of the terminals ${ }^{29}$. In another study, three central S cone bipolar cell axon terminals were completely reconstructed from serial sections, but gap junctions were not described ${ }^{33}$. Central and peripheral retina may differ in this respect. Another electron microscopic study of area centralis in cat retina identified a single on cone bipolar cell type (b5) that was neither directly nor indirectly coupled to AII amacrine cells and that had a morphology similar to that of the $\mathrm{S}$ cone bipolar cell ${ }^{28}$. However, conclusions regarding this cell type were generalized from a single b5 cell.

In the rabbit retina, the connexin-36 gap-junction permeant tracer Neurobiotin labeled putative S cone bipolar cells when injected into AII amacrine cells (personal communication, S. Mills, University of Texas Medical School). Combined with our findings, this suggests that rod input to the $\mathrm{S}$ cone pathway is a general feature of the mammalian visual system.

\section{Night vision may utilize most visual pathways}

Does the visual system utilize all of the fibers of the optic nerve for scotopic vision or does it reserve some fibers for cone signals to reduce rod-cone signal mixing and the corresponding deleterious consequences for color vision? Many studies have highlighted the challenges faced by the visual system at night: a sparse collection of absorbed photons must be detected on a background of substantial cellular and synaptic noise ${ }^{22}$. This, combined with anatomical and physiological observations that AII amacrine cells form synapses with many cone bipolar cell types ${ }^{28,34,35}$, suggests that most or all RGC types may participate in scotopic vision. However, several physiological studies have suggested that only a fraction of RGC types may participate in low scotopic vision $7,8,18,24$.

In peripheral primate retina, it appears that rod signals diverge to many and perhaps all RGC types. Previous anatomical and physiological studies have suggested that rods contribute input via the AII pathway to peripheral parasol cells ${ }^{7,36}$, midget cells ${ }^{37,38}$, giant sparse (melanopsin) cells ${ }^{39}$ and upsilon cells (G.D.F. and E.J.C., unpublished observations). The present results reveal rod input to SBCs. Furthermore, rod signals are provided to SBCs by gap junctions between AII amacrine cells and $\mathrm{S}$ cone bipolar cells. Therefore, large bistratified cells in the primate retina should also receive rod input from the AII amacrine pathway, because $S$ cone bipolar cells are thought to provide presynaptic input to both SBCs and large bistratified cells ${ }^{40,41}$. In sum, rod input via the AII pathway has been observed for each RGC type in the peripheral primate retina that has been studied under scotopic conditions.

It remains unclear whether these results will generalize to the central primate retina ${ }^{7}$ and whether there are major differences in the divergence of AII amacrine cell signals to various RGC types in primates versus other mammals. Perhaps surprisingly, SBCs exhibited a sensitivity under low scotopic conditions that was qualitatively similar to that of on parasol cells (Fig. 3). However, our results do not quantitatively compare the signal-to-noise properties of SBCs to those of on parasol cells or to those of other RGC types, a comparison 
that will determine which RGC types provide the most reliable signals to the brain when photons are scarce.

\section{Rod input to SBCs may explain blue bias in night vision}

The observation that scenes appear bluish at mesopic light levels dates to the nineteenth century ${ }^{42}$. Furthermore, psychophysical experiments have shown that rod activation biases color judgments toward blue hues $^{6}$ and influences color discriminations involving changes in S cone activation $^{43,44}$. Models explaining these results postulate rod input to $\mathrm{SBCs}^{45,46}$; however, as described above, previous physiological experiments have largely failed to observe such input.

The light levels at which the perceptual effects of rod activation on $\mathrm{S}$ cone signals are maximized are $2-10 \mathrm{Td}^{46}$, corresponding to $\sim 60-300 \mathrm{P}^{\star}$ per rod per s. These values roughly match the light levels for which the opponent spectral tuning of SBCs was most influenced by rod activation (Fig. 6). Thus, the present results may help to explain the psychophysical results. Activation of the rods, similarly to activation of $S$ cones, results in an increased spike rate in SBCs. Thus, to the degree that SBC spikes contribute to the perception of blue, rod activation would be expected to produce a bias toward the perception of blue.

\section{METHODS}

Methods and any associated references are available in the online version of the paper at http://www.nature.com/natureneuroscience/.

Note: Supplementary information is available on the Nature Neuroscience website.

\section{ACKNOWLEDGMENTS}

We thank C.K. Hulse for technical assistance; M.I. Grivich, D. Petrusca, W. Dabrowski, A. Grillo, P. Grybos, P. Hottowy and S. Kachiguine for technical development; H. Fox, M. Taffe, E. Callaway and K. Osborn for providing access to retinas; $\mathrm{H}$. Wässle and J. O'Brien for providing antibodies; and S. Barry for machining. We thank the San Diego Supercomputer Center and the National Science Foundation (Cooperative Agreements 05253071 and 0438741) for large-scale data storage. This work was supported by the Helen Hay Whitney Foundation (G.D.F.), Deutsche Forschungsgemeinschaft (M.G.), a US National Institutes of Health National Research Service Award (NS054519-01), the Chapman Foundation (J.L.G.), the Miller Institute for Basic Research in Science, the University of California at Berkeley (J.S.), a Burroughs Wellcome Fund Career Award at Scientific Interface (A.S.), the McKnight Foundation (A.M.L. and E.J.C.), National Science Foundation grant PHY-0750525 (A.M.L.), a Sloan Research Fellowship and US National Institutes of Health grants EY13150 (E.J.C.), EY06472 (D.W.M.) and EY10608 (D.W.M.).

\section{AUTHOR CONTRIBUTIONS}

G.D.F., D.W.M. and E.J.C. conceived the experiments and wrote the manuscript. G.D.F., M.G., J.L.G., J.S., A.S. and E.J.C. performed the electrophysiological experiments. C.R. and D.W.M. carried out the immunolabeling experiments. A.S. and A.L.M. provided and supported the large-scale multi-electrode array system.

Published online at http://www.nature.com/natureneuroscience/.

Reprints and permissions information is available online at http://npg.nature.com/ reprintsandpermissions/.

1. Masland, R.H. The fundamental plan of the retina. Nat. Neurosci. 4, 877-886 (2001).

2. Dacey, D. Origins of perception: retinal ganglion cell diversity and the creation of parallel visual pathways. in The Cognitive Neurosciences (ed. Gazzaniga, M.) 281-301 (MIT Press, Cambridge, Massachusetts, 2004).

3. Field, G.D. \& Chichilnisky, E. Information processing in the primate retina: circuitry and coding. Annu. Rev. Neurosci. 30, 1-30 (2007).

4. Dacey, D.M. \& Lee, B. The 'blue-on' opponent pathway in primate retina originates from a distinct bistratified ganglion cell type. Nature 367, 731-735 (1994).

5. Szmajda, B.A., Grunert, U. \& Martin, P. Retinal ganglion cell inputs to the koniocellular pathway. J. Comp. Neurol. 510, 251-268 (2008).

6. Buck, S. Rod-cone interactions in human vision. in The Visual Neurosciences (eds Chalupa, L. M. \& Werner, J. S.) 863-878 (MIT Press, Cambridge, Massachusetts, 2004).

7. Lee, B.B., Smith, V., Pokorny, J. \& Kremers, J. Rod inputs to macaque ganglion cells. Vision Res. 37, 2813-2828 (1997).

8. Wiesel, T.N. \& Hubel, D. Spatial and chromatic interactions in the lateral geniculate body of the rhesus monkey. J. Neurophysiol. 29, 1115-1156 (1966).
9. Virsu, V. \& Lee, B. Light adaptation in cells of macaque lateral geniculate nucleus and its relation to human light adaptation. J. Neurophysiol. 50, 864-878 (1983).

10. Litke, A.M. et al. What does the eye tell the brain? Development of a system for the large scale recording of retinal output activity. IEEE Trans. Nucl. Sci. 51, 1434-1440 (2004).

11. Field, G.D. et al. Spatial properties and functional organization of small bistratified ganglion cells in primate retina. J. Neurosci. 27, 13261-13272 (2007).

12. Kolb, H. \& Famiglietti, E. Rod and cone pathways in the inner plexiform layer of cat retina. Science 186, 47-49 (1974).

13. Bloomfield, S.A. \& Dacheux, R. Rod vision: pathways and processing in the mammalian retina. Prog. Retin. Eye Res. 20, 351-384 (2001).

14. Chichilnisky, E.J. \& Baylor, D. Receptive-field microstructure of blue-yellow ganglion cells in primate retina. Nat. Neurosci. 2, 889-893 (1999).

15. Rodieck, R. The First Steps in Seeing (Sinauer, Sunderland, Massachusetts, 1998).

16. Sharpe, L.T. \& Stockman, A. Rod pathways: the importance of seeing nothing. Trends Neurosci. 22, 497-504 (1999).

17. Gouras, P. \& Link, K. Rod and cone interaction in dark-adapted monkey ganglion cells. J. Physiol. (Lond.) 184, 499-510 (1966).

18. Cleland, B.G. \& Levick, W. Properties of rarely encountered types of ganglion cells in the cat's retina and an overall classification. J. Physiol. (Lond.) 240, 457-492 (1974).

19. Barlow, H.B., Fitzhugh, R. \& Kuffler, S. Change of organization in the receptive fields of the cat's retina during dark adaptation. J. Physiol. (Lond.) 137, 338-354 (1957).

20. Troy, J.B., Bohnsack, D. \& Diller, L. Spatial properties of the cat x-cell receptive field as a function of mean light level. Vis. Neurosci. 16, 1089-1104 (1999).

21. Peichl, L. \& Wassle, H. The structural correlate of the receptive field centre of alpha ganglion cells in the cat retina. J. Physiol. (Lond.) 341, 309-324 (1983).

22. Field, G.D., Sampath, A. \& Rieke, F. Retinal processing near absolute threshold: from behavior to mechanism. Ann. Rev. Physiol. 67, 491-514 (2005).

23. Hornstein, E.P., Verweij, J., Li, P. \& Schnapf, J. Gap-junctional coupling and absolute sensitivity of photoreceptors in macaque retina. J. Neurosci. 25, 11201-11209 (2005).

24. Völgyi, B., Deans, M., Paul, D. \& Bloomfield, S. Convergence and segregation of the multiple rod pathways in mammalian retina. J. Neurosci. 24, 11182-11192 (2004).

25. Slaughter, M.M. \& Miller, R. 2-amino-4-phosphonobutyric acid: a new pharmacological tool for retina research. Science 211, 182-185 (1981).

26. Müller, F., Wassle, H. \& Voigt, T. Pharmacological modulation of the rod pathway in the cat retina. J. Neurophysiol. 59, 1657-1672 (1988).

27. Murphy, G.J. \& Rieke, F. Network variability limits stimulus-evoked spike timing precision in retinal ganglion cells. Neuron 52, 511-524 (2006).

28. Cohen, E. \& Sterling, P. Demonstration of cell types among cone bipolar neurons of cat retina. Phil. Trans. R. Soc. Lond. B 330, 305-321 (1990).

29. Marshak, D.W., Aldrich, L., Del Valle, J. \& Yamada, T. Localization of immunoreactive cholecystokinin precursor to amacrine cells and bipolar cells of the macaque monkey retina. J. Neurosci. 10, 3045-3055 (1990).

30. Wässle, H., Grunert, U., Chun, M. \& Boycott, B. The rod pathway of the macaque monkey retina: identification of All-amacrine cells with antibodies against calretinin. J. Comp. Neurol. 361, 537-551 (1995).

31. Mills, S.L., O'Brien, J., Li, W., O'Brien, J. \& Massey, S. Rod pathways in the mammalian retina use connexin 36. J. Comp. Neurol. 436, 336-350 (2001).

32. Lyubarsky, A.L., Daniele, L. \& Pugh, E.J. From candelas to photoisomerizations in the mouse eye by rhodopsin bleaching in situ and the light-rearing dependence of the major components of the mouse erg. Vision Res. 44, 3235-3251 (2004).

33. Calkins, D.J., Tsukamoto, Y. \& Sterling, P. Microcircuitry and mosaic of a blue-yellow ganglion cell in the primate retina. J. Neurosci. 18, 3373-3385 (1998).

34. Veruki, M.L. \& Hartveit, E. Electrical synapses mediate signal transmission in the rod pathway of the mammalian retina. J. Neurosci. 22, 10558-10566 (2002)

35. Petrides, A. \& Trexler, E. Differential output of the high-sensitivity rod photoreceptor: All amacrine pathway. J. Comp. Neurol. 507, 1653-1662 (2008).

36. Jacoby, R.A. \& Marshak, D. Synaptic connections of db3 diffuse bipolar cell axons in macaque retina. J. Comp. Neurol. 416, 19-29 (2000).

37. Dunn, F.A., Lankheet, M. \& Rieke, F. Light adaptation in cone vision involves switching between receptor and post-receptor sites. Nature 449, 603-606 (2007).

38. Grünert, U. Anatomical evidence for rod input to the parvocellular pathway in the visual system of the primate. Eur. J. Neurosci. 9, 617-621 (1997).

39. Dacey, D.M. et al. Melanopsin-expressing ganglion cells in primate retina signal colour and irradiance and project to the Ign. Nature 433, 749-754 (2005).

40. Dacey, D.M. \& Packer, O. Colour coding in the primate retina: diverse cell types and cone-specific circuitry. Curr. Opin. Neurobiol. 13, 421-427 (2003).

41. Schein, S., Sterling, P., Ngo, L., Huang, T. \& Herr, S. Evidence that each s cone in macaque fovea drives one narrow-field and several wide-field blue-yellow ganglion cells. J. Neurosci. 24, 8366-8378 (2004)

42. von Kries, J. \& Nagel, W. Über den einfluss von lichtstärke und adaptation auf das sehen des dichromaten (Grünblinden). Zeitschrift für Psychologie und Physiologie der Sinnesorgane 12, 1-36 (1896).

43. Knight, R., Buck, S., Fowler, G. \& Nguyen, A. Rods affect s-cone discrimination on the farnsworth-munsell 100-hue test. Vision Res. 38, 3477-3481 (1998).

44. Cao, D., Zele, A. \& Pokorny, J. Chromatic discrimination in the presence of incrementa and decremental rod pedestals. Vis. Neurosci. 25, 399-404 (2008).

45. Buck, S.L., Knight, R. \& Bechtold, J. Opponent-color models and the influence of rod signals on the loci of unique hues. Vision Res. 40, 3333-3344 (2000).

46. Cao, D., Pokorny, J., Smith, V. \& Zele, A. Rod contributions to color perception: linear with rod contrast. Vision Res. 48, 2586-2592 (2008). 


\section{ONLINE METHODS}

Physiology. Retinas were obtained and recorded as described previously ${ }^{11}$. Briefly, eyes were enucleated from terminally anesthetized macaque monkeys (Macaca mulatta and Macaca fascicularis) that were used in the course of other experiments ${ }^{11}$, in accordance with guidelines for the care and use of animals set by the Institutional Animal Care and Use Committee of the Salk Institute. Immediately after enucleation, the anterior portion of the eye and vitreous were removed in room light. Following a dark-adaptation period of $>40 \mathrm{~min}$ at $32-33{ }^{\circ} \mathrm{C}$, segments of peripheral retina that were well attached to the pigment epithelium were isolated and placed flat, RGC layer down, on a planar array of 512 extracellular microelectrodes covering a $1,800 \mu \mathrm{m} \times 900 \mu \mathrm{m}$ region. During recording, isolated retinas were kept at $33-35{ }^{\circ} \mathrm{C}$ and were perfused with Ames' solution (Sigma-Aldrich) bubbled with 95\% $\mathrm{O}_{2}$ and $5 \% \mathrm{CO}_{2}$ ( $\mathrm{pH}$ 7.4). We added $10 \mu \mathrm{M} \mathrm{L}-\mathrm{APB}$ to the Ames' solution for some experiments (Fig. 4b-d). The L-APB solution was washed into or out of the recording chamber for $\sim 5 \mathrm{~min}$ before data collection resumed. In experiments designed to test the reversibility of receptive field size and SBC response properties at mesopic conditions (Figs. 2d and 6), the retina was left attached to the pigment epithelium and choroid and recorded at $31-35{ }^{\circ} \mathrm{C}$.

Recordings were analyzed offline to isolate the spikes of different cells, as described previously ${ }^{11}$. Briefly, candidate spike events were detected using a threshold on each electrode, and the voltage waveform on the center and nearby electrodes in the vicinity of spike events was extracted. Spikes were clustered on the basis of waveform, and spike clusters were identified as candidate neurons if they exhibited a refractory period and an average spike rate $>0.25 \mathrm{~Hz}$. Duplicate recordings of the same cell were identified by temporal cross-correlation and removed.

Light calibration and stimuli. An optically reduced stimulus from a gammacorrected cathode ray tube computer display (Sony Multiscan E100) refreshing at $120 \mathrm{~Hz}$ was focused on the photoreceptor outer segments. Light intensity was controlled by neutral density filters in the light path. The emission spectrum of each display primary was measured with a PR-701 spectra-radiometer (PhotoResearch) after passing through the optical elements between the display and the retina. The power of each display primary was measured at the preparation with a calibrated photodiode (UDT Instruments). For rods, the photo-isomerization rate $\left(\mathrm{P}^{*}\right.$ per rod per $\left.\mathrm{s}\right)$ was estimated by computing the inner product of the power-scaled emission spectra per unit area with the spectral sensitivity of macaque rhodopsin ${ }^{47}$ and multiplying by the effective collecting area of the primate rod $(1.2 \mu \mathrm{m})^{47}$.

The mean photo-isomerization rate for the $\mathrm{L}, \mathrm{M}$ and $\mathrm{S}$ cones at the photopic light levels (Fig. 1) was calculated in the same manner as described for the rods, with an estimated effective cone collecting area of $0.37 \mu \mathrm{m}^{48,49}$. These calculations yielded bleaching rates for the $\mathrm{L}, \mathrm{M}$ and $\mathrm{S}$ cones of 800,800 and $440 \mathrm{P}^{\star}$ per cone per s, respectively, at the photopic light level.

In experiments with the pigment epithelium attached to the retina, the visual stimulus passed through the mostly transparent electrode array and the retina before being absorbed in the photoreceptor outer segments. The emission spectra of the display primaries and their emission powers were calibrated as described above, but for a different optical path. At the highest mean light level presented, the bleaching rates for the $\mathrm{L}, \mathrm{M}$ and $\mathrm{S}$ cones and the rods were $1,400,1,200$ and $400 \mathrm{P}^{\star}$ per cone per $\mathrm{s}$ and $3,000 \mathrm{P}^{\star}$ per rod per $\mathrm{s}$, respectively (Fig. 6). In these experiments, the predicted relative sensitivities to the red, green and blue display primaries for the rods was 0.070, 1.0 and 0.84 (normalized to the green primary), respectively. For the L, M and S cones, the predicted relative sensitivities to the red, green and blue primaries were 0.41 , 1.0 and $0.18,0.15,1.0$ and 0.29 , and $0.02,0.11$ and 1.0 , respectively (the latter normalized to the blue primary).

These estimates of the rod and cone photo-isomerization rates did not correct for the angle of illumination and pigment self screening in the photoreceptor outer segments because the precise angle of illumination and the amount of bleached pigment were unknown. In addition, estimates of pigment density of the rods and cones vary by roughly a factor of 2. However, an assumption of axial rather than transverse illumination and a pigment density of 0.17 (ref. 48$)(0.37)^{47}$ for the cones (rods) changed the estimated photo-isomerization rates by $<50 \%$.
In some experiments (Figs. 3 and 4), light steps were presented as stimuli. These step stimuli were spatially uniform and periodic, changing from one light level to another every $3 \mathrm{~s}$ (Fig. 4) or $5 \mathrm{~s}$ (Fig. 3). The stimuli cycled through gray, white, gray, black and back to gray. The average light level was determined by the intermediate (gray) value of the step sequence, where the gamma corrected display was set to $50 \%$ of its maximum output. When the display was set to nominal black, the output was reduced 130-fold compared with gray. The photon flux of the white step was twice that of the gray.

Receptive field characterization. A white noise stimulus composed of a lattice of squares (pixels), each flickering randomly and independently at $120 \mathrm{~Hz}$ ( $30 \mathrm{~Hz}$ in some experiments) was used to characterize the spatiotemporal response properties of recorded $\mathrm{RGCs}^{50}$. Across experiments, the sizes of individual pixels varied from $58 \mu \mathrm{m}$ to $116 \mu \mathrm{m}$ on a side. The intensity of each display primary at each pixel location was chosen from a binary distribution at each refresh. At photopic light levels, the stimulus was RGB white noise; the three display primaries at each pixel location varied independently of one another. The contrast of this stimulus for each of the three display primaries was $96 \%$ (difference between the maximum and minimum intensities divided by the sum of intensities). In some experiments at scotopic light levels, an RGB stimulus was used. In other experiments, the stimulus was black and white; the three display primaries were modulated together at each pixel location with a contrast of $96 \%$. The black-and-white stimulus generated a higher effective contrast because the display primaries were modulated synchronously. This allowed receptive fields of equal signal-to-noise ratios to be estimated in less time, but this stimulus does not provide spectral information about the RGC light responses. Control analyses indicated that the black-and-white stimulus did not introduce a bias in receptive field size estimates compared with the RGB stimulus. Typically, STAs were calculated from either 15- or 30-min presentations of the white-noise stimulus.

SBC receptive fields were summarized by fitting with a parametric model. The model consisted of the product of two profiles: spatial and temporal ${ }^{11}$. The temporal profile was a difference of lowpass filters. The spatial profile consisted of a two-dimensional elliptical Gaussian function. This model was sufficient to describe the receptive fields measured with black-and-white whitenoise stimuli under scotopic conditions. For scotopic presentations of the RGB stimulus, fits included two additional parameters that described the relative sensitivity to contrast modulation of the three display primaries. Photopic stimuli always consisted of RGB white noise, and only the spatial and temporal profiles of the blue display primary were fitted. This fit accurately described the $\mathrm{S}$ cone-mediated receptive field center of $\mathrm{SBCs}^{11}$. Several parameters of the fits were extracted to visualize receptive field extent: the location of the Gaussian fit center, the s.d. along the major and minor axes and the angle of the major axis. These parameters defined an ellipse for each cell that represented the 1-s.d. contour of the Gaussian fit. In the figures, receptive field outlines are represented using this contour.

Cell type classification and identification. The morphological type of recorded cells was determined using a two step procedure, as described previously ${ }^{11}$. Briefly, cells were first grouped into functional classes on the basis of lightresponse properties. Functional classes were identified by clustering according to response properties (Fig. 1b,g). At the high light level (Fig. 1b), two response properties distinguished SBCs from all other on type RGCs: the ratio of the peak amplitudes of the STA time courses to the green and blue monitor primaries, and the receptive field radius. The receptive field radius was given by the radius of a circle with an area equal to that defined by the 1-s.d. contour of the two-dimensional Gaussian fit to the spatial profile of the STA. At the low light level (Fig. 1g), SBCs were functionally classified on the basis of the receptive field radius and the weights associated with the first principal component of the STA time courses. These weights were obtained by performing principal components analysis on the concatenated STA time courses associated with the red, green and blue display primaries. Correspondences between functional classes and morphological types were determined by cell density and light-response properties. This procedure definitively identified the SBCs in each recording ${ }^{11}$.

Electrophysiological image. After the spikes from a given neuron were isolated on a source electrode, the average electrical activity in a time window from 
$0.5 \mathrm{~ms}$ before to $3 \mathrm{~ms}$ after the spike was calculated across the electrode array ${ }^{10}$. Because the spiking activity of any given cell is largely independent of all other cells, the average electrical activity across the array reveals a unique electrical footprint for every cell, reflecting its position, extent of dendritic arbor and axon trajectory relative to the electrode array. Electrophysiological images are displayed as a dot pattern, where each dot represents the location of the electrode at which the signal from the neuron was detected and the diameter of the dot is proportional to the amplitude of the peak of the electrical waveform associated with a spike, collapsed over time (Fig. 1c-e,h-j).

The uniqueness of the electrophysiological image for a given cell across an experiment was confirmed by calculating the correlation coefficient between the electrophysiological image of a reference cell in recording $\mathrm{A}$ and the electrophysiological images of all the cells in recording B (for example, between high and low light levels). Accumulated across all (53) SBCs in three representative preparations, the electrophysiological image with the highest correlation coefficient in recording B was always given by a cell that was a member of the functional class that formed a mosaic with a conserved spatial organization to that observed in recording $\mathrm{A}$, and whose receptive field overlapped with the reference cell in A. The uniqueness of the electrophysiological image allowed cells to be tracked from one recording condition to another.

Anatomy. Two Macaca mulata and one Macaca fascicularis were killed with an overdose of pentobarbital, following a protocol approved by the Animal Welfare Committee of the University of Texas Health Science Center at Houston. The eyes were removed, the anterior halves were discarded and the vitreous humor was removed from the posterior half with fine forceps. For the M. mulata, pieces of the eyecup were fixed with $4 \%$ paraformaldehyde (wt/vol) in $0.1 \mathrm{M}$ sodium phosphate buffer ( $\mathrm{pH} 7.4)$ overnight at $4{ }^{\circ} \mathrm{C}$. The retinas were isolated and rinsed with phosphate-buffered saline (PBS). For the M. fascicularis, the retina was fixed for $2 \mathrm{~h}$ at $20{ }^{\circ} \mathrm{C}$ with $0.1 \%$ glutaraldehyde (vol/vol) added to the primary fixative. It was then treated with $1 \% \mathrm{NaBH}(\mathrm{wt} / \mathrm{vol})$ in PBS for $60 \mathrm{~min}$. The retinas were embedded in agarose and $50-70-\mu \mathrm{m}$ vertical sections were cut with a VT1000S microtome (LEICA). The sections were incubated for $14 \mathrm{~d}$ at $4{ }^{\circ} \mathrm{C}$ in PBS (pH 7.4) with 5\% Chemiblock (vol/vol, Millipore) and $0.3 \%$ Triton X-100 (vol/vol, Sigma). Goat antibody to calretinin (1:1,000, Chemicon), rabbit antibody to G6-gly $(1: 1,000)$ and mouse monoclonal antibody to connexin 36 (1:1,000, Chemicon) were used as primary antibodies. The tissue was then incubated for $2 \mathrm{~h}$ at $20{ }^{\circ} \mathrm{C}$ in PBS with $5 \%$ Chemiblock and $0.3 \%$ Triton X-100 with the following affinity-purified secondary antibodies raised in donkeys: Cy3 antibody to goat $\operatorname{IgG}(1: 1,000$, Jackson ImmunoResearch), Alexa 488 antibody to rabbit IgG (1:1,000, Molecular Probes) and Cy5 antibody to mouse IgG (1:500, Jackson ImmunoResearch). The images were acquired using a Zeiss LSM 510 confocal laser scanning microscope. Illustrations were prepared using LSM (Zeiss) and Photoshop 6.0 (Adobe) software.

47. Baylor, D.A., Nunn, B. \& Schnapf, J. The photocurrent, noise and spectral sensitivity of rods of the monkey macaca fascicularis. J. Physiol. (Lond.) 357, 575-607 (1984).

48. Baylor, D.A., Nunn, B. \& Schnapf, J. Spectral sensitivity of cones of the monkey macaca fascicularis. J. Physiol. (Lond.) 390, 145-160 (1987).

49. Schnapf, J.L., Nunn, B., Meister, M. \& Baylor, D. Visual transduction in cones of the monkey macaca fascicularis. J. Physiol. (Lond.) 427, 681-713 (1990).

50. Chichilnisky, E.J. A simple white noise analysis of neuronal light responses. Network 12 199-213 (2001). 